Résumés des conférences et travaux

\title{
Érudition historique et philologique de l'âge classique aux Lumières
}

Jean-Louis Quantin

\section{OpenEdition}

\section{Journals}

Édition électronique

URL : https://journals.openedition.org/ashp/1196

DOI : 10.4000/ashp.1196

ISSN : 1969-6310

\section{Éditeur}

Publications de l'École Pratique des Hautes Études

\section{Édition imprimée}

Date de publication : 1 octobre 2011

Pagination : 225-228

ISSN : 0766-0677

\section{Référence électronique}

Jean-Louis Quantin, «Érudition historique et philologique de l'âge classique aux Lumières », Annuaire de l'École pratique des hautes études (EPHE), Section des sciences historiques et philologiques [En ligne], 142 | 2011, mis en ligne le 23 septembre 2011, consulté le 06 juillet 2021. URL : http:// journals.openedition.org/ashp/1196; DOI : https://doi.org/10.4000/ashp.1196 


\title{
ÉRUDITION HISTORIQUE ET PHILOLOGIQUE DE L’ÂGE CLASSIQUE AUX LUMIÈRES
}

\author{
Directeur d'études : M. Jean-Louis QuANTIN
}

Programme de l'année 2009-2010 : Érudition et négation de l'histoire : autour du P. Hardouin.

La conférence s'est penchée à deux reprises sur la question des faux fabriqués aux $\mathrm{XVI}^{\mathrm{e}}$ et $\mathrm{XVII}^{\mathrm{e}}$ siècles et sur le rôle, considérable, qu'ils tinrent dans le développement et la formalisation de la méthode critique : d'abord pour donner une vue d'ensemble sur « Faux et faussaires dans l'Europe moderne », en 2002-2003, puis, en 2007-2008, dans le cadre du commentaire suivi d'un inédit de Mabillon, le Memoire touchant les fables dont on a gasté l'histoire Ecclesiastique et Monastique d'Espagne depuis 80 ans $^{1}$. L'année 2009-2010 a été consacrée au revers du problème, si l'on ose dire, à savoir au pyrrhonisme historique dont le jésuite français Jean Hardouin (1646-1729) constitua la figure extrême et scandaleuse. On pourrait croire qu'il s'agissait là d'une aberration, d'un cas de délire voire de folie, n'étaient, d'une part, la forte intégration d'Hardouin dans la République des Lettres et les larges échos donnés à ses ouvrages dans les périodiques savants, d'autre part les polémiques très vives, plusieurs fois relancées, sur le lien éventuel entre ses vues et le projet politico-religieux de la Compagnie de Jésus. La démarche suivie a donc consisté à envisager ensemble le " système » du P. Hardouin, qui, en soi, n'est qu'une version érudite de la théorie du complot, et la réception d'Hardouin par ses contemporains, qui a précipité, au sens chimique du terme, des préoccupations et des inquiétudes essentielles de la culture du temps.

Il n'existe pas de monographie sur Hardouin, qui, comme il a été observé, « mérite un biographe d'une érudition, d'un latin, et d'un esprit sans pareils $»^{2}$, et la plupart des études qui lui ont été consacrées au $\mathrm{XX}^{\mathrm{e}}$ siècle ne vont pas au-delà d'une paraphrase de ses ouvrages imprimés - ou même, plus exactement, des analyses dont ceux-ci avaient fait l'objet, en français, dans des journaux comme la Bibliothèque choisie ou la Bibliothèque raisonnée des ouvrages des savants de l'Europe. Le travail d'interprétation historique avait été ouvert par R. R. Palmer, qui avait mis en évidence un courant catholique de défense de la tradition par le pyrrhonisme et bien montré le lien entre cette entreprise et la controverse janséniste. Il avait cependant moins insisté sur Hardouin que sur Berruyer, qui peut passer d'une certaine manière pour son disciple

1. Voir respectivement Livret-Annuaire de l'École pratique des Hautes Études, Section des Sciences historiques et philologiques, t. 18, 2002-2003, p. 276-279, et Annuaire de l'École pratique des Hautes Études, Section des Sciences historiques et philologiques, t. 140, 2007-2008, p. 323-325.

2. Alan Charles Kors, Atheism in France, 1650-1729, Princeton, NJ, 1990, note 69 p. 344 : « The extraordinary Hardouin deserves a biographer of unsurpassed erudition, Latinity, and wit ». 
dans la Compagnie de Jésus, mais n'a pas du tout le même rapport à l'érudition ${ }^{1}$. Cette ligne d'interprétation a été reprise, brièvement mais avec beaucoup de densité, par Bruno Neveu, qui est allé droit à l'essentiel : « Le Père Hardouin, effrayé des progrès de la religion de l'Antiquité chez les théologiens, cherche à établir sur un pyrrhonisme historique poussé à ses ultimes limites une Église libérée de l'Histoire $»^{2}$. Ont seuls depuis lors apporté du nouveau Catherine Northeast - sur la diffusion souterraine des vues d'Hardouin parmi les jésuites français, d'après les archives de la Compagnie $^{3}$-, et Anthony Grafton - sur la place d'Hardouin dans la tradition de l'antiquariat, en utilisant largement ses manuscrits à la Bibliothèque nationale de France ${ }^{4}$.

On a commencé par retracer les différentes phases de la carrière du P. Hardouin. Une première période, qu'on peut dire classique, s'inscrit nettement dans la continuité de l'érudition jésuite française du premier $\mathrm{XVII}^{\mathrm{e}}$ siècle : travaux numismatiques, édition en 1684 des discours de Thémistius, publication de l'édition de Théodoret préparée par le P. Garnier, édition de Pline l'Ancien en 1685 dans la collection ad usum Delphini, lancement d'une nouvelle collection conciliaire, sous le patronage de l'Assemblée du clergé de France. En 1689, son édition commentée de la lettre du pseudo-Chrysostome à Césaire montre que, s'il tend à prendre systématiquement le contre-pied des opinions courantes, il est encore très loin du pyrrhonisme : il défend l'authenticité du texte et s'efforce de montrer qu'il ne contredit en rien la doctrine catholique de la transsubstantiation. La cassure, au témoignage d'Hardouin lui-même dans Ad Censuram Scriptorum Veterum Prolegomena (Londres, 1766), date de 1690 : il commença en août de cette année à suspecter que tous les écrits d'Augustin étaient des faux et étendit ses soupçons en novembre à l'ensemble de la littérature patristique. Il est vraisemblable que cette conversion au pyrrhonisme explique la manière dont, au printemps suivant, il fut soudainement démis de son poste de régent de théologie, à l'étonnement des contemporains (voir la lettre de Daniel Larroque à Bayle du 23 avril 1691). Il reçut alors la charge moins exposée de bibliothécaire du collège Louis-leGrand, qu'il occupa jusqu'à sa mort, en 1729.

La seconde phase de la carrière d'Hardouin fut celle où il s'efforça, moyennant quelques ménagements rhétoriques, de publier son système. Il commença par une Chronologiae ex Nummis antiquis restitutae prolusio de nummis Herodiadum (Paris, 1693), où il rejeta Flavius Josèphe comme un auteur supposé, contredisant à la fois le Nouveau Testament et les médailles, et rempli de gallicismes. À ce propos, dans un passage fameux, Hardouin exposa « la conjecture de quelqu'un dont les conjectures ne sont pas toujours absurdes mais qui, dans le cas présent, est peut-être plus soupçonneux qu'il ne convient », à savoir que toute l'histoire ancienne avait été inventée, «je ne sais il y a combien de temps », par une bande de faussaires, à partir des monnaies

1. R. R. Palmer, Catholics and Unbelievers in eighteenth century France, Princeton, NJ, 1939, chapitre 3, p. 53-76.

2. Bruno Neveu, «Archéolâtrie et modernité dans le savoir ecclésiastique au XVII ${ }^{\mathrm{e}}$ siècle », $X V I I^{e}$ siècle, $\mathrm{n}^{\circ} 131$ (avril-juin 1981), p. 180-183, repris dans Érudition et religion aux XVII et XVIII ${ }^{e}$ siècles, Paris, 1994, p. 377-379.

3. Catherine M. Northeast, The Parisian Jesuits and the Enlightenment, 1700-1762, Oxford, 1991.

4. Anthony Grafton, « Jean Hardouin: the antiquary as pariah », Journal of the Warburg and Courtauld Institutes, t. 62 (1999), p. 241-264. 
et d'un très petit nombre de textes authentiques (dont l'Historia naturalis de Pline l'Ancien). Dans la Chronologia veteris Testamenti, ad vulgatam versionem exacta, et nummis antiquis illustrata (Paris, 1697), Hardouin identifia - de manière cryptée mais sans qu'on puisse s'y tromper - le patron de ces faussaires comme l'empereur Frédéric II. Il soutint en outre que la Vulgate latine était le seul texte authentique de l'Écriture, beaucoup plus ancien que les textes grec et hébreu actuellement conservés : tous les anciens auteurs qui ne s'accordaient pas avec la Vulgate étaient donc sans autorité.

L'un et l'autre ouvrage furent supprimés par ordre des supérieurs jésuites, et s'attirèrent des réfutations. Le scandale n'éclata cependant vraiment qu'avec l'intervention de Mathurin Veyssière La Croze, un ancien bénédictin de Saint-Maur converti au protestantisme et établi à Berlin, dont l'intéressante carrière a été parfaitement retracé en 2001 par un collègue allemand ${ }^{1}$. Ce fut en 1707, avec ses Dissertations historiques sur divers sujets, qu'il inaugura la campagne, anti-hardouiniste et anti-jésuite à la fois, qui devait l'occuper jusqu'à la fin de ses jours : montrant - avec raison - que derrière l'Antiquité profane, c'étaient les Pères de l'Église qui étaient visés, il affirma que « l'ouvrage du P. Hardouin [était] celui de toute la Société ». Cette dénonciation fut reprise en latin l'année suivante. L'annonce, juste après, qu'allait paraître à Amsterdam un recueil in-folio d'Opera selecta d'Hardouin (reprenant en particulier ses ouvrages supprimés) contraignit la Compagnie de Jésus à prendre publiquement position. Les supérieurs parisiens firent paraître en 1709 une « Declaration » contre les Opera selecta, et obligèrent Hardouin à se rétracter. La troisième phase, dans les deux décennies suivantes, jusqu'à la mort du jésuite, le vit donc officiellement réduit au silence - du moins s'agissant de ses vues pyrrhonistes, car il fut autorisé à publier par ailleurs -, mais continuant à faire du prosélytisme auprès des jeunes jésuites, et, surtout, rédigeant à l'appui de ses thèses quantité de travaux manuscrits. La mort d'Hardouin ouvrit donc une dernière période, celle des publications posthumes : Opera varia (Amsterdam, 1733), Commentarius in Novum Testamentum (Amsterdam, 1741), Ad Censuram Scriptorum Veterum Prolegomena (Londres, 1766) - ce dernier texte donnant la clef de tout le système.

Un second temps de l'année a été consacré au commentaire suivi des deux principaux traités recueillis dans les Opera varia de 1733 : Athei detecti et Pseudo Virgilius. Dans Athei detecti, Hardouin soumet à une analyse serrée les ouvrages des principaux auteurs tant de l'augustinisme théologique (Jansénius, Pascal, Nicole, Quesnel) que du cartésianisme philosophique qui lui était souvent associé (Descartes, Malebranche), et entreprend de démontrer qu'ils ont tous enseigné l'athéisme. Hardouin met en fait en œuvre, en la poussant jusqu'à l'absurde, une herméneutique de type inquisitorial, tournée vers l'hérésie implicite et cachée. Ses accusations s'inscrivent en outre dans des débats théologiques très précis, ainsi sur l'intention du ministre des sacrements ou sur la forme du baptême. Sous couleur de dénoncer l'athéisme, Hardouin procède en

1. Martin Mulsow, Die drei Ringe. Toleranz und clandestine Gelehrsamkeit bei Mathurin Veyssière La Croze (1661-1739), Tübingen, 2001. Cet important ouvrage est entièrement passé sous silence dans le médiocre article de Didier Boisson, « Entre foi et doutes. Itinéraire de Mathurin Veyssière de La Croze, un mauriste converti au protestantisme à la fin du XVII ${ }^{\mathrm{e}}$ siècle », Revue d'histoire de l'Église de France, t. 94 (2008), p. 283-299. 
fait à une récapitulation de toute la polémique antijanséniste de la Compagnie de Jésus. Quant à Pseudo Virgilius, il s'agit d'un commentaire de l'Énéide pour montrer que l'ouvrage est un faux médiéval - Virgile n'ayant écrit, selon Hardouin, que les Bucoliques et les Géorgiques. L'argumentation mobilise une érudition considérable mais fait aussi écho aux débats contemporains sur la nature et les règles du poème épique, en particulier dans le cadre de la querelle d'Homère, à laquelle Hardouin avait luimême contribué, en 1716, par son Apologie d'Homere - un des rares auteurs anciens qu'épargnait son pyrrhonisme. L'hardouinisme, s'il s'enracine dans la crise janséniste, se rattache en même temps à la querelle des anciens et des modernes. Hardouin, qui parle très souvent le langage de l'antiquariat, peut aussi parler celui de la critique littéraire. On en a tiré pour les auditeurs une leçon méthodologique sur la nécessité d'éclairer systématiquement un texte par son intertexte. Ce qui pouvait passer pour une divagation se révèle d'une grande richesse dès lors que l'on saisit à qui-Perrault, $\mathrm{M}^{\mathrm{me}}$ Dacier, La Motte - Hardouin fait implicitement référence.

Le troisième et dernier temps de la conférence a porté sur le commentaire d'Hardouin sur Augustin, resté inédit et conservé dans vingt-neuf cahiers à la Bibliothèque nationale ${ }^{1}$. Le jésuite y applique au "pseudo-Augustin » (aussi désigné comme « le fripon »), en qui il voit « le chef de la cohorte impie », la même lecture inquisitoriale qui lui faisait trouver l'athéisme dans Descartes et dans Pascal. L'anti-augustinisme, constamment présent à l'arrière-plan du système, se trouve ici explicitement développé.

Cette conférence était la dernière que le directeur d'études donnait dans la salle d'égyptologie, où il enseignait depuis son entrée à la Section, à l'automne 2002. Il voudrait pouvoir accepter les événements avec autant de détachement qu'un de ses héros, comme disent les Anglais, Henry Dodwell, démis de sa chaire de Camdenian lecturer à Oxford en novembre 1691, et dont l'unique commentaire, en publiant ses cours l'année suivante, fut cette citation du livre de Job : Dominus dedit, Dominus abstulit : Sicut Domino placuit, ita factum est : sit nomen Domini benedictum.

1. Jean Hardouin, Censura librorum Augustini, Fonds Latin 2745-2767, imparfaitement décrits dans Catalogue général des manuscrits latins, III, Paris, 1952, p. 56-59. 